PREPARED FOR THE U.S. DEPARTMENT OF ENERGY, UNDER CONTRACT DE-AC02-76CH03073

PPPL-3863

PPPL-3863

UC-70

Strange Attractors in Drift Wave Turbulence

by

Jerome L.V. Lewandowski

September 2003

NM|

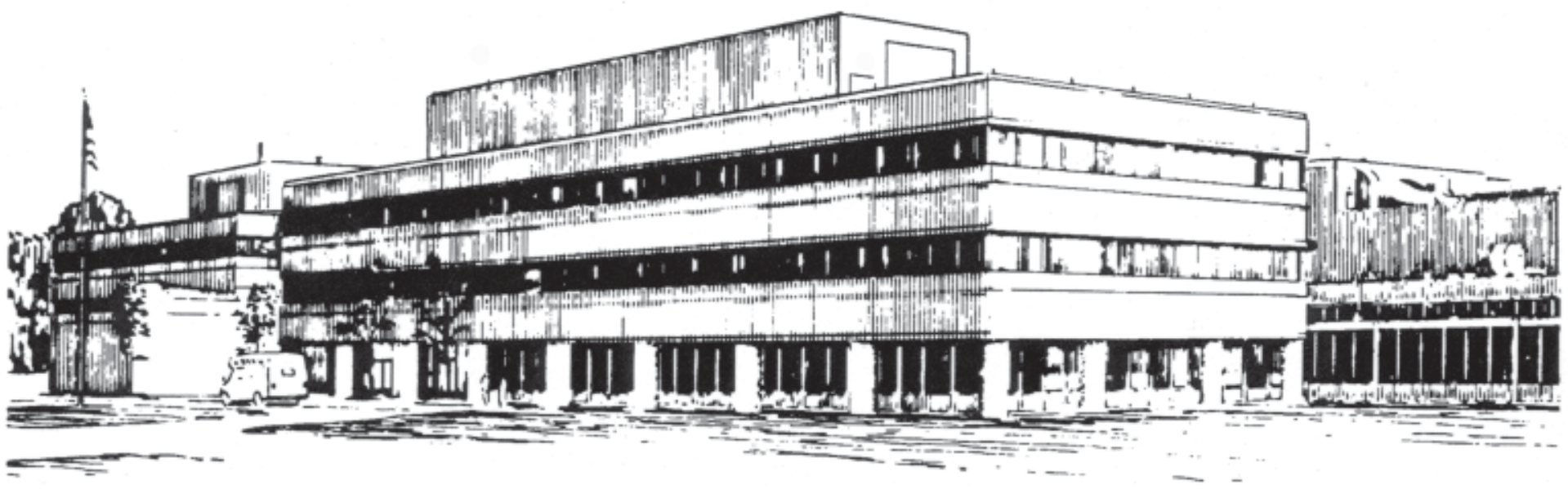

PRINCETON PLASMA PHYSICS LABORATORY PRINCETON UNIVERSITY, PRINCETON, NEW JERSEY 


\section{PPPL Reports Disclaimer}

This report was prepared as an account of work sponsored by an agency of the United States Government. Neither the United States Government nor any agency thereof, nor any of their employees, makes any warranty, express or implied, or assumes any legal liability or responsibility for the accuracy, completeness, or usefulness of any information, apparatus, product, or process disclosed, or represents that its use would not infringe privately owned rights. Reference herein to any specific commercial product, process, or service by trade name, trademark, manufacturer, or otherwise, does not necessarily constitute or imply its endorsement, recommendation, or favoring by the United States Government or any agency thereof. The views and opinions of authors expressed herein do not necessarily state or reflect those of the United States Government or any agency thereof.

\section{Availability}

This report is posted on the U.S. Department of Energy's Princeton Plasma Physics Laboratory Publications and Reports web site in Fiscal Year 2003. The home page for PPPL Reports and Publications is: http://www.pppl.gov/pub_report/

DOE and DOE Contractors can obtain copies of this report from:

U.S. Department of Energy

Office of Scientific and Technical Information

DOE Technical Information Services (DTIS)

P.O. Box 62

Oak Ridge, TN 37831

Telephone: (865) 576-8401

Fax: (865) 576-5728

Email: reports@adonis.osti.gov

This report is available to the general public from:

National Technical Information Service

U.S. Department of Commerce

5285 Port Royal Road

Springfield, VA 22161

Telephone: $1-800-553-6847$ or

(703) $605-6000$

Fax: (703) 321-8547

Internet: http://www.ntis.gov/ordering.htm 


\title{
Strange Attractors in Drift Wave Turbulence
}

\author{
Jerome L.V. Lewandowski \\ Princeton University, Princeton Plasma Physics Laboratory, Princeton, NJ 08543
}

\section{Introduction}

There are growing experimental, numerical and theoretical evidences that the anomalous transport observed in tokamaks and stellarators is caused by slow, drift-type modes (such as trapped electron modes and ion-temperature gradient-driven modes). Although typical collision frequencies in hot, magnetized fusion plasmas can be quite low in absolute values, collisional effects are nevertheless important since they act as dissipative sinks. As it is well known, dissipative systems with many (strictly speaking more than two) degrees of freedom are often chaotic and may evolve towards a so-called attractor.

This paper shows that strange attractors in collisional, electrostatic drift wave turbulence with kinetic electrons can exist and that their fractal dimension are actually quite small; this result suggests the presence of deterministic dynamics with few key variables but displaying chaotic behavior (because of the fractal dimensionality of the attractor). Another important conclusion is that our observation of a low-dimensional attractor for this specific model of drift wave turbulence has been achieved using an accurate scheme for kinetic electrons (splitting scheme; see next section). In the presence of kinetic electrons, standard schemes (e.g. $\delta f$ scheme [2]) fail to resolve the underlying dynamics of the system, that is the fractal dimension cannot be measured.

\section{Splitting Scheme}

As mentioned in the Introduction, the measurement of the fractal dimension of the attractor in electrostatic drift wave turbulence has been made possible by using an accurate electron scheme. In order to stress the relevance of strange attractors to drift-wave turbulence, we consider a shearless slab model for electrostatic drift waves. We start from the collisionless, electrostatic, gyrokinetic Vlasov equation, in the long-wavelength limit, for particles species $j$ with mass $m_{j}$ and charge $q_{j}$

$$
\frac{d F_{j}}{d t} \equiv \frac{\partial F_{j}}{\partial t}+\left(v_{\|} \widehat{\mathbf{b}}_{0}+\mathbf{V}_{E}\right) \cdot \nabla F_{j}-\frac{q_{j}}{m_{j}} \widehat{\mathbf{b}}_{0} \cdot \nabla \Phi \frac{\partial F_{j}}{\partial v_{\|}}=C\left(F_{j}\right)
$$

where $\widehat{\mathbf{b}}_{0}=\mathbf{B}_{0} / B_{0}$ is a unit vector, $\mathbf{V}_{E}=c \widehat{\mathbf{b}}_{0} \times \nabla \Phi / B_{0}$ is the $\mathbf{E} \times \mathbf{B}$ drift velocity, and $C\left(F_{j}\right)$ is the collision operator. The confining magnetic field is taken to be of the form $\mathbf{B}_{0}=B_{0}(\widehat{\mathbf{z}}+\theta \widehat{\mathbf{y}})$ where $\theta$ is a small parameter, together with the simplification of $\partial / \partial z \mapsto k_{z} \equiv 0$. Collisional effects on the ion distribution are neglected, $C\left(F_{i}\right)=0$; the effects of electron-ion collisions can be represented by the number-conserving, energy-conserving Lorentz collision operator [1] including only pitch-angle scattering in the velocity space for the electrons

$$
C\left(F_{e}\right)=\frac{\nu_{e i}}{2} \frac{1}{\sin \zeta} \frac{\partial}{\partial \zeta}\left(\sin \zeta \frac{\partial F_{e}}{\partial \zeta}\right)
$$

where $\nu_{e i}=4 \pi n_{0} e^{4} \ln \Lambda / m_{e}^{2} V_{\text {the }}^{3}$ is the collision frequency and $\zeta=\cos ^{-1} v_{\|} /\left(v_{\|}^{2}+v_{\perp}^{2}\right)^{1 / 2}$. Although the standard $\delta f$ scheme [2] works well for the ion dynamics, an accuracy problem arises when the scheme is used to treat the electron dynamics. The origin of this accuracy problem is related to the fact that the bulk of the electrons do not interact with the low-frequency waves but may (and usually do) transfer noise if their dynamics is not treated accurately. Therefore, it is natural to separate the electrons into two groups (adiabatic and nonadiabatic) to reflect their different 
responses to the low-frequency waves. To do so, we write the distribution $F_{j}$ as [4]

$$
F_{j}=\exp \left(-\frac{q_{j} \Phi}{T_{j}}\right) F_{M j}+h_{j},
$$

where $F_{M j}$ is the Maxwellian distribution for particle species $j$ and $h_{j}$ is the nonadiabatic response. Substituting representation (3) in Eq.(1) and using the relations of $\left(\partial / \partial t+v_{\|} \widehat{\mathbf{b}}_{0} \cdot \nabla\right) F_{M j}=0$ and $\mathbf{V}_{E} \cdot \nabla \Phi \equiv 0$, we obtain an evolution equation for the nonadiabatic response

$$
\frac{d h_{j}}{d t}=C\left(F_{j}\right)+F_{M j} \exp \left(-\frac{q_{j} \Phi}{T_{j}}\right)\left(\boldsymbol{\kappa}_{j} \cdot \mathbf{V}_{E}+\frac{q_{j}}{T_{j}} \frac{\partial \Phi}{\partial t}\right)
$$

where $\kappa_{j}=\kappa\left[1-\frac{\eta_{j}}{2}\left(1-\bar{v}_{\|}^{2}\right)\right], \bar{v}_{\|}=v_{\|} / V_{t h j}$ and $\kappa=-\nabla n_{0} / n_{0}$. As it is evident from Eq.(4) the contribution due to the free streaming particles will not, unlike the corresponding $\delta f$ scheme, appear in the equation for the marker weight. The field equations related to $\Phi$ and $\partial \Phi / \partial t$ have been solved using a multigrid solver; the details are given in Ref. [4]. The initial loading of the markers in velocity space is carried out using a low-noise technique described in Ref. [5]. The linear properties of the splitting scheme [4] and the energy conservation properties have been presented elsewhere [3] and these results are not reproduced here.

\section{Characterization of Strange Attractors}

It is already an accepted notion that many nonlinear dissipative dynamical systems do not approach stationary or periodic states asymptotically. Instead, with appropriate values of their parameters, they tend towards strange attractors on which the motion is chaotic, i.e. not periodic and unpredictable over long times, being extremely sensitive on the initial conditions $[7,8,6]$. Typically a strange attractor arises when the flow in phase space does not contract a volume element in all directions, but stretches it in some. In order to remain confined to a bounded domain, the volume element gets folded at the same time, so that it has after some time a multisheeted structure [7,6]. In our model, dissipation through collisions is what allows for phase space contraction.

Grasseberger and Procaccia [9] have introduced an important measure of an attractor known as the correlation exponent, which is based on correlations between random points on the attractor. The basic idea behind the correlation exponent measure is that trajectories belonging to an attractor, although not dynamically correlated, are spatially correlated. Introducing the correlation integral $C(\ell)$ these authors have shown that, for small enough $\ell, C(\ell) \sim \ell^{\alpha}$, where $\alpha$ is the so-called correlation exponent. Grassberger and Procaccia have proved that the information dimension, $\sigma$, the Hausdorff dimension, $D$, and the correlation exponent, $\alpha$, satisfy the inequality

$$
\alpha \leq \sigma \leq D .
$$

In most cases, the inequality (5) is rather tight. To measure the spatial correlation of the attractor, Grassberger and Proccacia consider a time series $\left\{\mathbf{X}_{i} \equiv \mathbf{X}(t+i \Delta t) ; i=1, \cdots, M\right\}$ of points on the attractor, where $\Delta t$ is the (fixed) time step; they define the correlation integral [9] as

$$
C(\ell) \equiv \lim _{M \mapsto \infty} \frac{\widehat{M}(\ell)}{M^{2}},
$$

where

$$
\widehat{M}(\ell) \equiv \sum_{i j} H\left(\left|\mathbf{X}_{i}-\mathbf{X}_{j}\right|-\ell\right)
$$

is the number of pairs $(i, j)$ whose distance $d_{i j}=\left|\mathbf{X}_{i}-\mathbf{X}_{j}\right|$ is less than $\ell$; in Eq.(7) $H(x)$, denotes the Heaviside function. One important conclusion of the work by Grassberger and Proccacia is that, for small $\ell$, the correlation integral $C(\ell)$ grows like a power

$$
C(\ell) \sim l^{\alpha},
$$


and that this correlation exponent $(\alpha)$ can be taken as a measure of the local structure of a strange attractor [9]. The usefulness of this measure for a system with many degrees of freedom is highlighted in the next section.

\section{Numerical Results}

The implementation of the Grassberger-Procaccia algorithm has been tested against known results for the (one-dimensional non-invertible) logistic map [8] and the (two-dimensional invertible) map [11]. In both cases the measured correlation exponent provides a close lower bound to the Hausdorff dimension [10].

Having tested the implementation of the Grassberger-Proccacia algorithm, we consider the case of fully developed electrostatic drift wave turbulence. Since there is no explicit source of dissipation (no phase space contraction) for the ion population, we measure the correlation exponent of the electron dynamics only. We randomly select a set of $M$ electron markers from the electron distribution function. Each sample $\mathbf{X}_{q}=\left(x_{k}^{(n)}, v_{\| k}^{(n)}\right)$ is recorded for each marker $k$ at time step $n$. In order to prevent spurious spatial correlations, the system must be in the fully nonlinear state; in this paper, the positions in phase space $\mathbf{X}_{q}$ were recorded for $\omega_{c i} t \geq 3000$ (fully turbulent regime) for $N_{s}$ time steps. The distance in phase space between $\mathbf{X}_{q}$ and $\mathbf{X}_{q^{\prime}}$ is simply given by

$$
d_{q, q^{\prime}}=\left|\mathbf{X}_{q}-\mathbf{X}_{q^{\prime}}\right|=\left\{\left[x_{k}^{(n)}-x_{k^{\prime}}^{\left(n^{\prime}\right)}\right]^{2}+\left[v_{\| k}^{(n)}-v_{\| k^{\prime}}^{\left(n^{\prime}\right)}\right]^{2}\right\}^{1 / 2}
$$

and the correlation integral is computed as in Eq.(6). In a typical simulation, both the number of sampling markers $M$ and the number of time steps $N_{s}$ is varied to ensure convergence. Figure 1 (left) shows the electron correlation integral, $C_{e}(\ell)$, as a function of $\ell / \ell_{0}$, where $\ell_{0}$ is arbitrary; the collision frequency is $\nu_{e i}=10^{-4}$. For very small distances, the data for $C_{e}(\ell)$ deviate from a power law, but that was to be expected: the values of $\mathbf{X}_{q}$ and $\mathbf{X}_{q^{\prime}}$ are strongly correlated. For larger $\ell$ the correlation integral follows a power law over 7 orders of magnitude. The $\chi^{2}$ fit yields a correlation exponent of $\alpha=0.0126$. This means that the low-dimensional attractor is somewhere between a point $(D=0)$ and a line $(D=1)$. Since the system has many degrees of freedom, such a low-dimensional may seem surprising; however, for a very different physical system, Nicolis and Nicolis [12] have found a strange attractor with a small dimension $D$ in a system with many degrees of freedom (see next section) The key factor here is the rate of phase space contraction.
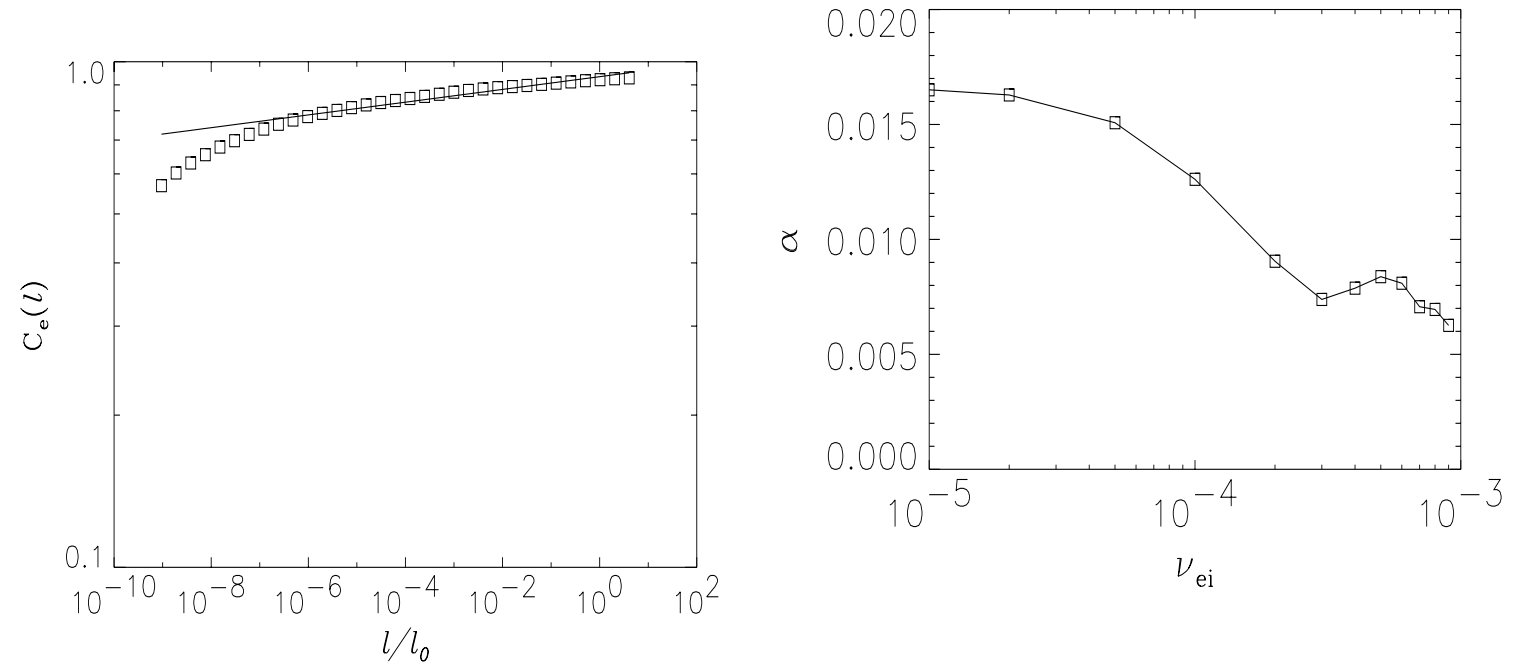

Figure 1: Correlation integral for $\nu_{e i}=10^{-4}$ as a function of $\ell / \ell_{0}$ (left); dependence of $C(\ell)$ on $\nu_{e i}$.

To pursue this argument, we have measured the dependence of the correlation exponent $\alpha$ on the collision frequency (Figure 1; right). The general trend is a decrease in the correlation 
exponent, and therefore a decrease in the Hausdorff dimension, with increasing collision frequency. This is not surprising as the phase space contraction rate is related to, but not necessarily directly proportional to, the collision frequency.

\section{Conclusions}

We have identified the existence of a low-dimensional strange attractor in particle-in-cell, electrostatic drift-wave turbulence. The dimension of the attractor has been estimated based on the measurement of the correlation exponent [9] (a lower bound to the usual Hausdorff dimension). It has been shown that the dimension of the attractor is sensitive to the electron-ion collision frequency since this quantity is related to the contraction rate in phase.

Numerical results have shown the presence of a low-dimensional attractor in a system with many degrees of freedom. In a different context, Nicolis and Nicolis [12] have studied the attractor associated with the climatic evolution over the past million years based on isotope records of deepsea cores. The surprising result of Nicolis and Nicolis's work is that, although the climate has very many degrees of freedom, a well-defined low-dimensional attractor was identified based on the experimental time series. Their results and our results suggest that some physical systems with many degrees of freedom can possess low-dimensional attractors, implying the presence of deterministic dynamics with few key variables but displaying unpredictable behavior (because of the fractal dimensionality of the attractor).

As a final remark, we note that, since the Grassberger-Procaccia algorithm is based on the information contained in one (or many) time series, their method can be useful to analyze and characterize strange attractors from experimental measurements in fusion plasmas.

\section{Acknowledgments}

This research was supported by Contract No DE-AC02-76CH03073 and the Scientific Discovery through Advanced Computing (SciDAC) initiative (U.S. Department of Energy).

\section{References}

[1] R.D. Hazeltine and F.L. Hinton, Rev. Mod. Phys. 48, 239 (1976).

[2] R.E. Denton and M. Kotschenreuther, J. Comp. Phys. 119, 283 (1995).

[3] J.L.V. Lewandowski, Plasma Phys. Control. Fusion, 45, L39 (2003).

[4] J.L.V. Lewandowski, Phys. Plasmas, 10(8), 3204 (2003).

[5] J.L.V. Lewandowski, Phys. Letters A, 313, 291 (2003).

[6] D. Ruelle and F. Takens, Commun. Math. Phys. 20, 167 (1971).

[7] E.N. Lorenz, J. Atmos. Sci. 20, 130 (1963).

[8] R.M. May, Nature 261, 459 (1976).

[9] P. Grassberger and I. Procaccia, Phys. Rev. Lett., 50, 346 (1983).

[10] D.A. Russel, J.D. Hanson and E. Ott, Phys. Rev. Lett., 45, 1175 (1980).

[11] M. Henon, Commun. Math. Phys., 50, 69 (1976).

[12] C. Nicolis and G. Nicolis, Nature, 311, 529 (1984). 


\section{External Distribution}

Plasma Research Laboratory, Australian National University, Australia

Professor I.R. Jones, Flinders University, Australia

Professor João Canalle, Instituto de Fisica DEQ/IF - UERJ, Brazil

Mr. Gerson O. Ludwig, Instituto Nacional de Pesquisas, Brazil

Dr. P.H. Sakanaka, Instituto Fisica, Brazil

The Librarian, Culham Laboratory, England

Mrs. S.A. Hutchinson, JET Library, England

Professor M.N. Bussac, Ecole Polytechnique, France

Librarian, Max-Planck-Institut für Plasmaphysik, Germany

Jolan Moldvai, Reports Library, Hungarian Academy of Sciences, Central Research Institute for Physics, Hungary

Dr. P. Kaw, Institute for Plasma Research, India

Ms. P.J. Pathak, Librarian, Institute for Plasma Research, India

Ms. Clelia De Palo, Associazione EURATOM-ENEA, Italy

Dr. G. Grosso, Instituto di Fisica del Plasma, Italy

Librarian, Naka Fusion Research Establishment, JAERI, Japan

Library, Laboratory for Complex Energy Processes, Institute for Advanced Study, Kyoto University, Japan

Research Information Center, National Institute for Fusion Science, Japan

Dr. O. Mitarai, Kyushu Tokai University, Japan

Dr. Jiangang Li, Institute of Plasma Physics, Chinese Academy of Sciences, People's Republic of China

Professor Yuping Huo, School of Physical Science and Technology, People's Republic of China

Library, Academia Sinica, Institute of Plasma Physics, People's Republic of China

Librarian, Institute of Physics, Chinese Academy of Sciences, People's Republic of China

Dr. S. Mirnov, TRINITI, Troitsk, Russian Federation, Russia

Dr. V.S. Strelkov, Kurchatov Institute, Russian Federation, Russia

Professor Peter Lukac, Katedra Fyziky Plazmy MFF UK, Mlynska dolina F-2, Komenskeho Univerzita, SK-842 15 Bratislava, Slovakia

Dr. G.S. Lee, Korea Basic Science Institute, South Korea

Institute for Plasma Research, University of Maryland, USA

Librarian, Fusion Energy Division, Oak Ridge National Laboratory, USA

Librarian, Institute of Fusion Studies, University of Texas, USA

Librarian, Magnetic Fusion Program, Lawrence Livermore National Laboratory, USA

Library, General Atomics, USA

Plasma Physics Group, Fusion Energy Research Program, University of California at San Diego, USA

Plasma Physics Library, Columbia University, USA

Alkesh Punjabi, Center for Fusion Research and Training, Hampton University, USA

Dr. W.M. Stacey, Fusion Research Center, Georgia Institute of Technology, USA

Dr. John Willis, U.S. Department of Energy, Office of Fusion Energy Sciences, USA

Mr. Paul H. Wright, Indianapolis, Indiana, USA 
The Princeton Plasma Physics Laboratory is operated by Princeton University under contract with the U.S. Department of Energy.

\author{
Information Services \\ Princeton Plasma Physics Laboratory \\ P.O. Box 451 \\ Princeton, NJ 08543
}

Phone: 609-243-2750

Fax: 609-243-2751

e-mail: pppl_info@pppl.gov

Internet Address: http://www.pppl.gov 